\title{
The value of genomic relationship matrices to estimate levels of inbreeding
}

\author{
Beatriz Villanueva ${ }^{1 *}$, Almudena Fernández , María Saura ${ }^{1}$, Armando Caballero², Jesús Fernández', \\ Elisabeth Morales-González ${ }^{1}$, Miguel A. Toro ${ }^{3}$ and Ricardo Pong-Wong ${ }^{4}$
}

\begin{abstract}
Background: Genomic relationship matrices are used to obtain genomic inbreeding coefficients. However, there are several methodologies to compute these matrices and there is still an unresolved debate on which one provides the best estimate of inbreeding. In this study, we investigated measures of inbreeding obtained from five genomic matrices, including the Nejati-Javaremi allelic relationship matrix $\left(F_{N E}\right)$, the Li and Horvitz matrix based on excess of homozygosity $\left(F_{L \& H}\right)$, and the VanRaden (methods 1, $F_{V R 1}$, and 2, $\left.F_{V R 2}\right)$ and Yang $\left(F_{Y A N}\right)$ genomic relationship matrices. We derived expectations for each inbreeding coefficient, assuming a single locus model, and used these expectations to explain the patterns of the coefficients that were computed from thousands of single nucleotide polymorphism genotypes in a population of Iberian pigs.

Results: Except for $F_{N E \text {, }}$ the evaluated measures of inbreeding do not match with the original definitions of inbreeding coefficient of Wright (correlation) or Malécot (probability). When inbreeding coefficients are interpreted as indicators of variability (heterozygosity) that was gained or lost relative to a base population, both $F_{N E J}$ and $F_{L \& H}$ led to sensible results but this was not the case for $F_{V R 1}, F_{V R 2}$ and $F_{Y A N}$. When variability has increased relative to the base, $F_{V R 1}, F_{V R 2}$ and $F_{Y A N}$ can indicate that it decreased. In fact, based on $F_{Y A N}$ variability is not expected to increase. When variability has decreased, $F_{V R 1}$ and $F_{V R 2}$ can indicate that it has increased. Finally, these three coefficients can indicate that more variability than that present in the base population can be lost, which is also unreasonable. The patterns for these coefficients observed in the pig population were very different, following the derived expectations. As a consequence, the rate of inbreeding depression estimated based on these inbreeding coefficients differed not only in magnitude but also in sign.

Conclusions: Genomic inbreeding coefficients obtained from the diagonal elements of genomic matrices can lead to inconsistent results in terms of gain and loss of genetic variability and inbreeding depression estimates, and thus to misleading interpretations. Although these matrices have proven to be very efficient in increasing the accuracy of genomic predictions, they do not always provide a useful measure of inbreeding.
\end{abstract}

\section{Background}

Inbreeding, i.e. the mating of individuals related by ancestry, is a fundamental concept in many areas of biology, including animal and plant breeding [1], human

*Correspondence: villanueva.beatriz@inia.es

${ }^{1}$ Departamento de Mejora Genética Animal, INIA, Ctra. de La Coruña, km 7.5, 28040 Madrid, Spain

Full list of author information is available at the end of the article

genetics [2, 3], and evolutionary [4] and conservation biology [5]. Inbreeding results in a reduction of genetic diversity, as it increases homozygosity at the expense of heterozygosity. This increase in homozygosity in turn increases the incidence of homozygous recessive defects and decreases population means for many quantitative traits (i.e., inbreeding depression), particularly those related to fitness $[6,7]$. 
The level of inbreeding of an individual is measured by the inbreeding coefficient, which was defined by Wright as the correlation between homologous alleles of the two gametes that unite to form the individual [8], and later by Malécot as the probability that two homologous alleles at a given locus are identical-by-descent [9]. The inbreeding coefficient also gives the proportion by which the heterozygosity of an individual is reduced by inbreeding [10] and, thus, the proportional loss of genetic variation. Classically, the inbreeding coefficient of an individual has been determined based on its pedigree. However, the pedigree-based inbreeding coefficient provides only expected proportions of the genome that are identical-by-descent.

The level of inbreeding has also been estimated from molecular data, such as those contained in high-density single nucleotide polymorphism (SNP) arrays. Genomic inbreeding coefficients can be more accurate than pedigree-based measures because they capture the variation due to Mendelian sampling and therefore can differentiate among individuals with the same pedigree (e.g. [11]). Genomic measures also allow us to differentiate inbreeding at specific regions of a genome, which is not possible with pedigree-based inbreeding.

Several methods have been proposed to calculate inbreeding coefficients using genomic data, including methods based on continuous runs of homozygosity (e.g. $[11,12]$ and methods applied on a SNP-by-SNP basis (e.g. [13-16]). Some of the latter measures come from matrices that are used to obtain genomic predictions in animal breeding. In this context, best linear unbiased predicted (BLUP) evaluations are replaced by genomic BLUP (GBLUP) evaluations, in which the numerator relationship matrix (NRM) is substituted by one of several genomic relationship matrices (GRM) [15, 16]. Given that the diagonals of the NRM equal 1 plus the inbreeding coefficients for the corresponding individuals, it has been generally accepted that the diagonals of the GRM are 1 plus the realized inbreeding level for the corresponding individuals. These genomic measures of inbreeding have been widely used [11, 17-47]. However, they can result in very different outcomes and the correlations between these estimators vary greatly and can even be negative, e.g. [27, 35]. Thus, there is still an unresolved debate on which are the best measures of inbreeding.

In this study, we compared genomic inbreeding coefficients that were obtained from different SNP-by-SNP methods to understand their relationship with traditional definitions of inbreeding. First, we describe different coefficients based on genomic information at the individual level. Second, we derive expectations at the population level for the different coefficients based on a single locus model. These expectations are then used to explain the patterns of the coefficients computed based on thousands of SNP genotypes across the genome in a highly inbred pig population.

\section{Methods}

Inbreeding coefficients obtained from genomic data

Individual inbreeding coefficients were obtained from the diagonal elements of five different genomic relationship matrices. These coefficients have been widely used in the literature, but under different names (see Table 1) and there is no consensus about the nomenclature. Here, the name chosen for each coefficient

Table 1 Summary of the names given to different genomic inbreeding coefficients in the literature

\begin{tabular}{|c|c|c|}
\hline $\begin{array}{l}\text { Nomenclature used } \\
\text { in this paper }\end{array}$ & $\begin{array}{l}\text { Nomenclature used } \\
\text { in the literature }\end{array}$ & References \\
\hline \multirow[t]{8}{*}{$F_{N E J}$} & $F_{\mathrm{PH}}$ & [19] \\
\hline & $F_{M}$ & {$[20]$} \\
\hline & $F_{\mathrm{MOL}}$ & [33] \\
\hline & Homozygosity & [21] \\
\hline & $F_{\mathrm{HOM}}$ & {$[28,35]$} \\
\hline & $\mathrm{HOM}_{\mathrm{SNP}}$ & {$[37]$} \\
\hline & SNP-Similarity* & [29] \\
\hline & $\mathrm{SIM}^{*}$ & [47] \\
\hline \multirow[t]{9}{*}{$F_{L \& H}$} & $F_{\mathrm{h}}$ or $F_{\mathrm{H}}$ & {$[11,25,40]$} \\
\hline & $F_{\text {snp }}$ & [26] \\
\hline & $F_{\text {HOM }}$ & {$[27,33,36,41,42,46,55]$} \\
\hline & $F_{\text {EXHOM }}$ & {$[35]$} \\
\hline & $F_{\text {PLINK }}$ & [31] \\
\hline & $F_{15}$ & {$[45]$} \\
\hline & $F_{\mathrm{EH}}$ & [34] \\
\hline & LHR & [22] \\
\hline & $L \& H^{*}$ & {$[47]$} \\
\hline \multirow[t]{5}{*}{$F_{V R 1}$} & $F_{\mathrm{GRM}}$ & {$[19,41]$} \\
\hline & $F_{\text {GRM1 }}$ & {$[35]$} \\
\hline & $F_{\mathrm{VR}}$ & [34] \\
\hline & $F_{G}$ & [17] \\
\hline & $\mathrm{VR} 1^{*}$ & [47] \\
\hline \multirow[t]{4}{*}{$F_{V R 2}$} & Fhatl, $F^{\prime}$ & {$[18,42,54]$} \\
\hline & $F_{\mathrm{GRM}}$ & {$[27,33]$} \\
\hline & $\mathrm{F}_{\mathrm{GRM} 2}$ & {$[35]$} \\
\hline & $\mathrm{VR} 2^{*}$ & [47] \\
\hline \multirow[t]{7}{*}{$F_{Y A N}$} & Fhatlll, $I^{\prime \prime \prime}$ & {$[18,42,54]$} \\
\hline & $F_{\mathrm{alt}}$ & {$[11,40]$} \\
\hline & GRM_F, FGRM & {$[21,28,31]$} \\
\hline & $F_{U N I}$ & {$[27,35,36,41,55]$} \\
\hline & $F_{\mathrm{grm}}$ & [31] \\
\hline & SNP-Yang* & [29] \\
\hline & YAN* & [47] \\
\hline
\end{tabular}

${ }^{*}$ Self-relationship or self-coancestry 
makes reference to the authors who first proposed or formulated it explicitly, to the best of our knowledge. We compared the following coefficients:

1. $F_{N E J}$ : inbreeding coefficient computed from the diagonal elements of the allelic relationship matrix of Nejati-Javaremi et al. [14] as:

$$
F_{N E J}=\frac{\sum_{k=1}^{S}\left(\sum_{i=1}^{2} \sum_{j=1}^{2} I_{i j_{k}}\right) / 2}{S}-1,
$$

where $I_{i j_{k}}$ is the identity of the two alleles $(i$ and $j)$ of the individual at SNP $k$, which takes the value of 1 if the two alleles are identical and 0 if they are not. Note that $F_{N E J}$ is simply the proportion of SNPs that are homozygous for the individual and thus it does not distinguish between identity-by-state (IBS) and identity-by-descent (IBD) [48].

2. $F_{L \& H}$ : inbreeding coefficient based on the relationship matrix that describes deviations from HardyWeinberg proportions, computed as:

$$
F_{L \& H}=\frac{S F_{N E J}-\sum_{k=1}^{S}\left[1-2 p_{k(0)}\left(1-p_{k(0)}\right)\right]}{S-\sum_{k=1}^{S}\left[1-2 p_{k(0)}\left(1-p_{k(0)}\right)\right]},
$$

where $p_{k(0)}$ is the frequency of the reference allele (allele $B$ ) of SNP $k$ in the base (reference) population [13]. $F_{L \& H}$ estimates the deviation of the observed frequency of homozygotes $(A A$ and $B B$ ) from that expected in the base population under Hardy-Weinberg proportions. Thus, it corrects for the homozygosity that was present in the base population and expresses molecular inbreeding in terms of IBD [42, $48,49]$.

3. $F_{V R 1}$ : inbreeding coefficient computed from the diagonal elements of the genomic relationship matrix obtained according to VanRaden's method 1 [15], as follows:

$$
F_{V R 1}=\frac{\sum_{k=1}^{S}\left(x_{k}-2 p_{k(0)}\right)^{2}}{2 \sum_{k=1}^{S} p_{k(0)}\left(1-p_{k(0)}\right)}-1,
$$

where $x_{k}$ is the genotype of the individual for SNP $k$, coded as 0,1 or 2 for genotypes $A A, A B$ and $B B$, respectively, and $p_{k(0)}$ is as defined for $F_{L \& H} . F_{V R 1}$ is based on the variance of additive genetic values and provides a measure relative to frequencies of the reference allele in the base population. However, $F_{V R 1}$ differs from $F_{L E H}$ in that with $F_{V R 1}$ homozygous genotypes are weighted by the inverse of their allele frequency and, thus, rare homozygous genotypes contribute more to the inbreeding measure than common homozygous genotypes [35].

4. $F_{V R 2}$ : inbreeding coefficient computed from the diagonal elements of the genomic relationship matrix obtained according to VanRaden's method 2 [15] as follows:

$$
F_{V R 2}=\frac{1}{S} \sum_{k=1}^{S} \frac{\left(x_{k}-2 p_{k(0)}\right)^{2}}{2 p_{k(0)}\left(1-p_{k(0)}\right)}-1,
$$

where $x_{k}$ and $p_{k(0)}$ are as for $F_{V R 1} . F_{V R 2}$ is similar to $F_{V R 1}$ but the summation across markers is made differently, such that the weight given to rare alleles is even greater. In $F_{V R 2}$, the contribution of each SNP is divided by its own variance, whereas in $F_{V R 1}$ the contributions of all SNPs are divided by the same denominator [35].

5. $F_{Y A N}$ : inbreeding coefficient computed from the diagonal elements of the genomic relationship matrix of Yang [16] as follows:

$$
F_{Y A N}=\frac{1}{S} \sum_{k=1}^{S} \frac{x_{k}^{2}-\left(1+2 p_{k(0)}\right) x_{k_{i}}+2 p_{k(0)}^{2}}{2 p_{k(0)}\left(1-p_{k(0)}\right)},
$$

where $x_{k}$ and $p_{k(0)}$ are as for $F_{V R 1}$. This coefficient is based on the correlation between uniting gametes $[16,42]$ and also gives more weight to homozygotes for the minor allele than to homozygotes for the major allele [40]. However, it has a lower sampling variance than the previous coefficients $[18,35]$ because it accounts for the sampling error associated with each SNP $[16,28]$.

The coefficients that depend on allele frequencies, i.e. $F_{L \& H}, F_{V R 1}, F_{V R 2}$, and $F_{Y A N}$, need to be computed using the initial frequencies; i.e. those in the base population. Note that $F_{N E J}$ is equivalent to $F_{V R 1}, F_{V R 2}$ and $F_{Y A N}$ when base population allele frequencies equal 0.5 [29].

\section{Expected genomic inbreeding coefficients at the population level: a single locus model}

Expected values for $F_{L \& H}, F_{V R 1}, F_{V R 2}$ and $F_{Y A N}$ at the population level were derived based on a single SNP model. Let $p_{(0)}$ be the frequency of allele $B$ in the base population. After $t$ generations, the frequency will have changed to $p_{(t)}$ due to random drift and selection, among other reasons. Assuming random mating, we can expect that genotype frequencies within a generation are in Hardy-Weinberg equilibrium. Thus, the expected $F$ for a group of individuals from generation $t$ can be obtained as:

$$
E(F)=\left[\text { freq }(\mathrm{AA}) F_{\mathrm{AA}}+\text { freq }(\mathrm{AB}) F_{\mathrm{AB}}+\text { freq }(\mathrm{BB}) F_{\mathrm{BB}}\right],
$$

where $\quad f r e q(\mathrm{AA})=\left(1-p_{(t)}\right)^{2}$, freq $(\mathrm{AB})=2 p_{(t)}\left(1-p_{t(t)}\right)$ and freq $(\mathrm{BB})=p_{(t)}^{2}$, and $F_{\mathrm{XY}}$ 
is the inbreeding coefficient for an individual with genotype $X Y$, which is computed using the initial frequency $p_{(0)}$, as described in the previous section. To assess the impact of initial and current allele frequencies on expected values of the evaluated inbreeding coefficients, the latter were evaluated for the whole range of values for $p_{(0)}$ and $p_{(t)}$.

\section{Evaluation of genomic inbreeding in a population of Guadyerbas pigs}

Results from the single locus model were evaluated in a population of Iberian pigs, with thousands of SNPs used to compute the inbreeding coefficients across the genome and at specific genomic regions.

\section{Pig samples and SNP genotypes}

The data used were from a herd of Guadyerbas Iberian pigs. The Guadyerbas strain is one of the most ancient surviving Iberian strains. It is highly inbred and in serious danger of extinction. The strain originated from four males and 20 females [50] and was conserved from 1944 until 2011 as a genetically isolated population. Accurate and complete genealogy was available from when the herd was first established (about 25 generations) and included 1178 animals born from 197 sires and 467 dams.

DNA samples were available for 86 males and 141 females born in the herd between 1992 and 2011 and were genotyped with the Illumina PorcineSNP60 BeadChip v1. SNP positions in the genome were based on the genome assembly Sscrofa 11.1. After quality control, as described in Saura et al. [20], 219 animals and 47,120 SNPs remained. In Iberian pigs, the generation interval is about three years, and thus for analysis of genomic inbreeding, we considered six cohorts of animals born in successive periods of three years, starting from year 1994 (Table 2).

\section{Patterns of genomic inbreeding coefficients}

Genomic coefficients were obtained for all genotyped pigs using the SNPs that segregated in cohort $1(17,951$ SNPs). The frequencies used to calculate $F_{L \& H}, F_{V R 1}$, $F_{V R 2}$, and $F_{Y A N}$ were those for cohort 1 (i.e. this cohort was considered to be the base population). Patterns of inbreeding across the genome were determined using sliding windows of 35 SNPs (average length of $4.25 \mathrm{Mb}$ ) that were moved one SNP at a time (17,339 windows). For each window, the average $F$ was computed in order to reduce the noisiness of single-locus estimates and to clarify the graphical representations [51-53]. For the coefficients that depend on allele frequencies $\left(F_{L \& H}, F_{V R 1}, F_{V R 2}\right.$ and $\left.F_{Y A N}\right)$, the formulae were applied within each window. Finally, values were averaged across individuals.
Table 2 Number of genotyped animals per cohort and sex in the Guadverbas population

\begin{tabular}{llcc}
\hline Cohort & Birth year range & Males & Females \\
\hline 1 & $1994-1996$ & 13 & 18 \\
2 & $1997-1999$ & 10 & 42 \\
3 & $2000-2002$ & 24 & 18 \\
4 & $2003-2005$ & 8 & 19 \\
5 & $2006-2008$ & 8 & 7 \\
6 & $2009-2011$ & 19 & 29 \\
Total & & 82 & 133 \\
\hline
\end{tabular}

\section{Inbreeding depression}

The behavior of the different genomic inbreeding coefficients will have consequences when they are used to estimate the rate of inbreeding depression across the genome. In order to investigate this, we performed a genome scan for inbreeding depression for the number of piglets born alive in the Guadyerbas population, using all genotyped sows with records born in the six cohorts (109 sows and 265 litter records) and the sliding window approach. The animals and phenotypic data used and the model fitted are described in detail in Saura et al. [26]. Briefly, inbreeding depression was estimated by regressing the number of piglets born alive on $F$ assuming a linear model. Fixed effects included the combination of season of farrowing and farrowing facilities, parity, strain of boar, and the linear regression on $F$. Random effects included additive genetic, permanent environmental, and residual effects. The variance-covariance matrix of additive genetic effects was assumed to be the pedigree-based numerator relationship matrix. Three measures of $F\left(F_{L \& H}, F_{V R 2}\right.$ and $\left.F_{Y A N}\right)$ computed using genotypes for all genotyped sows with phenotypic data born from cohort 1 to cohort 6, were used as covariates to estimate inbreeding depression.

\section{Results \\ Range of values and interpretation of the genomic inbreeding coefficients}

The inbreeding coefficients investigated differ in the range of values that they can contain and, with the exception of $F_{N E J}$, their ranges depend on the allele frequency in the base population $p_{(0)}$. Coefficient $F_{N E J}$ ranges from 0 to 1 because it is the proportion of homozygous SNPs. At the individual level, values for $F_{L \& H}$ range from $-\infty$ to 1 , and those for $F_{V R 1}, F_{V R 2}$ and $F_{Y A N}$ range from -1 to $\infty$ (Figs. 3,4 , and 5 , in Zhang et al. [27]). When all SNP genotypes are homozygous, $F_{L \& H}$ equals 1 and when all are heterozygous, it ranges 
from $-\infty$ to $-1 . F_{V R 1}$ and $F_{V R 2}$ cover the entire range (from -1 to $\infty$ ) both when all SNP genotypes are homozygous or heterozygous. Finally, when all SNP genotypes are homozygous, $F_{Y A N}$ ranges from 0 to $\infty$ and when they all are heterozygous, $F_{Y A N}$ equals -1 . Thus, values for $F_{L \& H}, F_{V R 1}, F_{V R 2}$ and $F_{Y A N}$ can be outside the permitted ranges for probabilities and correlations. Nevertheless, as inbreeding coefficients, they can still be interpreted as the proportional loss or gain in variability (heterozygosity) relative to the variability in the base population, with a negative value indicating that variability has been gained and a positive value that variability has been lost. It is also possible to gain more than $100 \%$ of the initial variability but it is not possible to lose more than $100 \%$. A value equal to 1 indicates that all the variability that was present in the base population has been lost but a value greater than 1 indicates that more variability than what existed initially has been lost, which does not make sense.

\section{Expected values of genomic inbreeding coefficients based on the single locus model}

Expected values for $F_{L \& H}, F_{V R 1}$ (or $F_{V R 2}$ ) and $F_{Y A N}$ based on the single locus model for the whole range of starting $\left(p_{(0)}\right)$ and current $\left(p_{(t)}\right)$ frequencies are shown in Fig. 1. Note that for a single locus model $E\left(F_{V R 1}\right)=E\left(F_{V R 2}\right)=E\left(F_{V R}\right)$.

The expected value for $F_{L \& H}$ (Fig. 1a) ranged from $-\infty$ and 1 . When the frequency of the minor allele increases (i.e. $\left.p_{(t)}>p_{(0)}\right)$ towards $0.5, E\left(F_{L \& H}\right)$ becomes negative, which indicates that some variability has been gained. This makes sense given that the maximum variability occurs when the frequency is 0.5 . Given that the upper limit of $E\left(F_{L \& H}\right)$ is 1 , when using this coefficient, one never expects more variability to be lost than the variability that initially existed. $E\left(F_{L \& H}\right)$ takes the value of 1 when the SNP becomes fixed, which is equivalent to all the variability being lost.

The expected value for $F_{V R}$ based on the diagonals of VanRaden's GRM is within the range $[0,1]$ for some combinations of $p_{(0)}$ and $p_{(t)}$, but for many other combinations it is outside this range (Fig. 1b). In fact, $E\left(F_{V R}\right)$ ranges from -1 to $\infty$. This means that $E\left(F_{V R}\right)$ can indicate that some variability has been gained but this gain can never be greater than $100 \%$ of the initial variability, as the lower limit is -1 . It also means that $E\left(F_{V R}\right)$ can indicate that more than $100 \%$ of the initial variability is lost, as it can take values higher than 1 (up to $\infty$ ).

In the right panel of Fig. 1b, the grid of initial and current frequencies is divided in regions where $E\left(F_{V R}\right)$ is $<0$, between 0 and 1 , or $>1$. When the frequency of the minor allele is doubled (i.e. $p_{(t)}=2 p_{(0)}$ ) but still lower than 0.5 , $E\left(F_{V R}\right)=1$, which means that $100 \%$ of the variability has been lost in the current generation when, in fact, variability has increased. For instance, if $p_{(0)}=0.25$ and $p_{(t)}=0.5$, $E\left(F_{V R}\right)$ indicates that all the initial variability has been lost, although the maximum variability is reached at a frequency of 0.5 . When the frequency of the minor allele more than doubles (i.e. $\left.p_{(t)}>2 p_{(0)}\right), E\left(F_{V R}\right)$ becomes $>1$ (for instance, for $p_{(0)}=0.1$ and $p_{(t)}=0.3, E\left(F_{V R}\right)=2.2$ ), which indicates that more than $100 \%$ of the initial variability has been lost, which is unreasonable. When the initial frequency of the minor allele is lower than 0.33 and decreases, then $E\left(F_{V R}\right)<0$, which indicates that variability has increased relative to its initial value. This is also the case when the minor allele is lost $\left(p_{(t)}=0\right)$. Thus, although variability in the current generation is lower than in the initial generation in these cases, $E\left(F_{V R}\right)$ incorrectly indicates that some variability has been gained.

On the one hand, although for a particular individual in the population, $F_{Y A N}$ can be negative (up to -1 ), contrary to $E\left(F_{V R}\right), E\left(F_{Y A N}\right)$ is never smaller than 0 (it ranges from 0 to $\infty$; Fig. 1c), which indicates that the level of heterozygosity cannot become larger than the level that existed initially, which is unreasonable. On the other hand, and as for $E\left(F_{V R}\right), E\left(F_{Y A N}\right)$ can be greater than 1, implying that more heterozygosity than what existed initially can be lost. In addition, although increasing the frequency of the minor allele towards 0.5 increases variability, $E\left(F_{Y A N}\right)$ can indicate a decrease in variability. For instance, when $p_{(0)}=0.1$ and remains at 0.1 in the current generation, $E\left(F_{Y A N}\right)=0$. However, if the frequency increases to $0.2, E\left(F_{Y A N}\right)$ becomes greater than $0\left(E\left(F_{Y A N}\right)=0.11\right)$, which indicates that some variability has been lost. And, if it increases to 0.5 (in theory a value at which the variability is maximum), $E\left(F_{Y A N}\right)$ becomes greater than $1\left(E\left(F_{Y A N}\right)=1.78\right)$, which indicates that more than $100 \%$ of the initial variability was lost.

When the initial frequency $\left(p_{(0)}\right)$ is set to 0.5 , the expected value for $F_{L \& H}, F_{V R}$ and $F_{Y A N}$ is the same regardless of the current frequency $\left(p_{(t)}\right)$ (see Additional file 1: Figure S1). In this scenario, these three coefficients range from 0 (when $p_{(t)}$ remains at 0.5 ) to 1 (when the SNP becomes fixed; i.e. $p_{(t)}=0$ or 1$)$.

Figure 2 shows the same profiles as in Fig. 1, but with the reference allele driven to a frequency of 0 (Fig. 2a), 0.5 (Fig. 2b) or 1 (Fig. 2c) in the current generation. Note that there is some redundancy in Fig. 2a, c since fixation of the major allele is equivalent to loss of the minor allele. For any value, $E\left(F_{L \& H}\right)=1$ when the SNP becomes fixed $\left(p_{(t)}=0\right.$ or 1$)$, regardless of the initial frequency, as expected (Fig. 2a, c). However, when the major allele is lost (Fig. 2a) or when the minor allele is fixed (Fig. 2c), both $E\left(F_{V R}\right)$ and $E\left(F_{Y A N}\right)$ take values greater than 1 (in fact their upper limit is $\infty)$. Losing the minor allele leads to negative values for $E\left(F_{V R}\right)$ when $p_{(0)}<1 / 3$ and its limit 

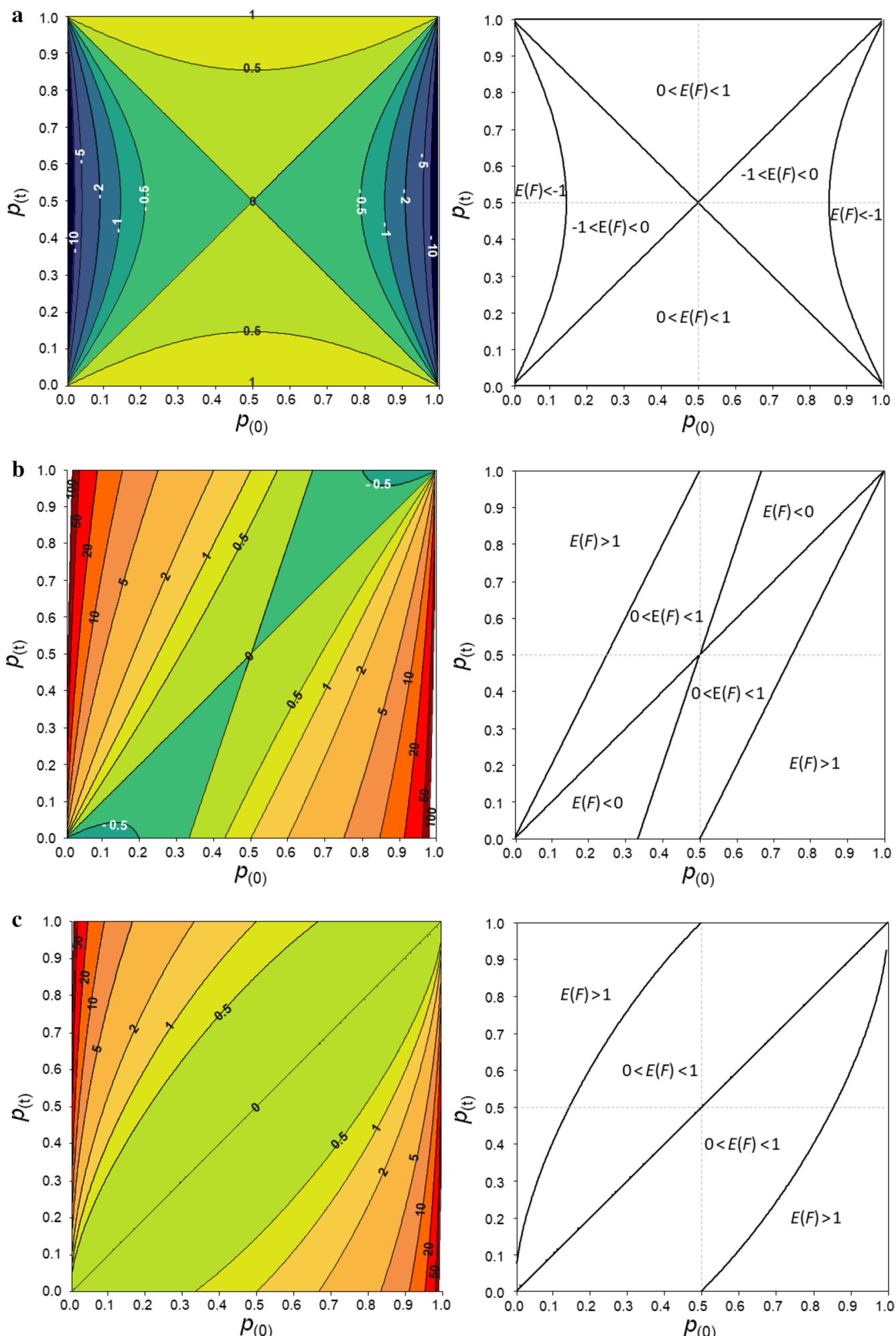

Fig. 1 Expected inbreeding coefficient based on excess of homozygosity $\left(F_{L \& H}\right)(\mathbf{a})$ and expected inbreeding coefficients computed from the diagonal elements of the genomic relationship matrices of VanRaden (methods 1 and $\left.2 ; F_{V R}=F_{V R 1}=F_{V R 2}\right)(\mathbf{b})$ and of Yang $\left(F_{Y A N}\right)(\mathbf{c})$ as a function of starting and current allele frequencies at a single locus. On the right, the grid of initial and current frequencies is divided in regions where the expected value of $F$ is $<-1,<0$, between -1 and 0 , between 0 and 1 , or $>1$ 

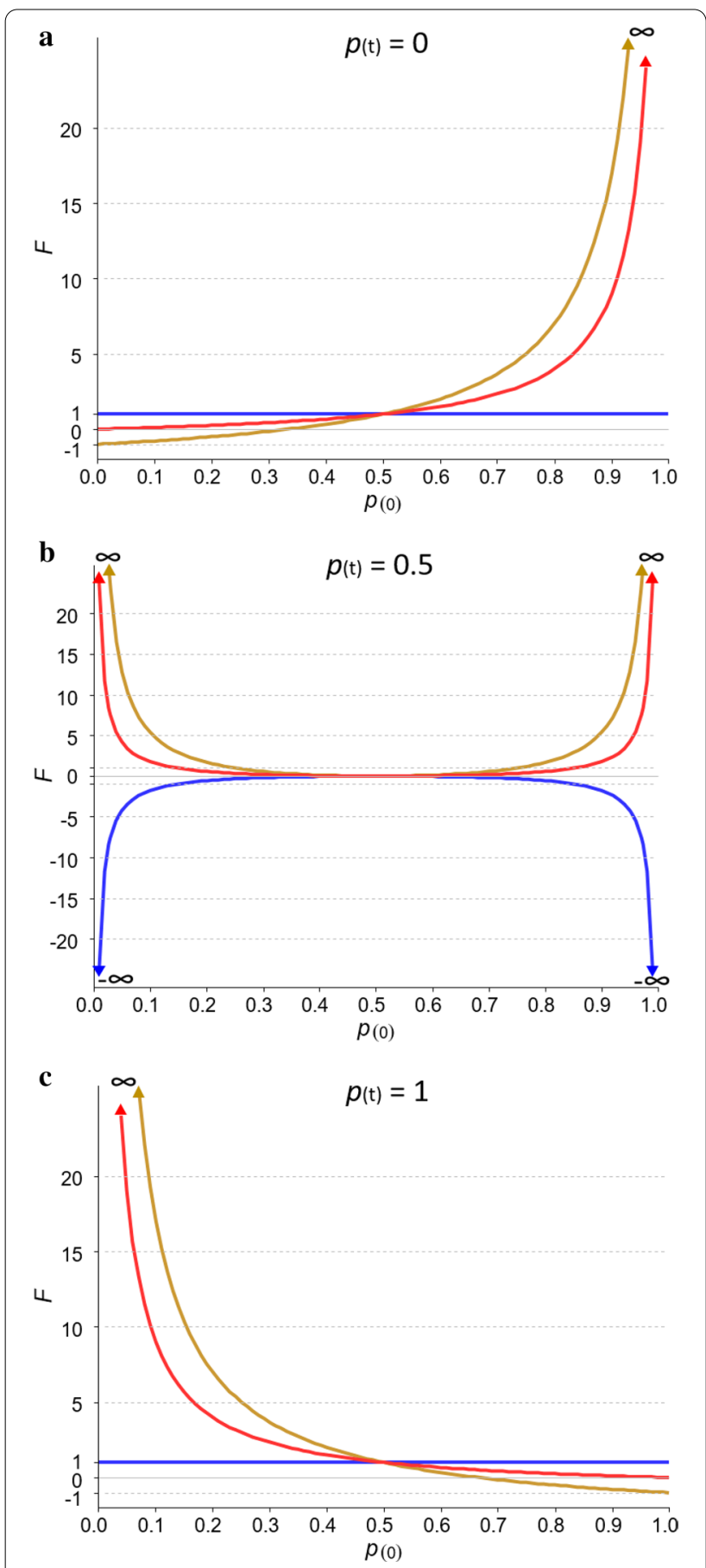

Fig. 2 Expected $F_{L \& H}, F_{V R}\left(F_{V R 1}=F_{V R 2}\right)$ and $F_{Y A N}$ in the generation in which the reference allele was lost $(\mathbf{a})$, driven to a frequency of 0.5 (b), or fixed (c) relative to the initial frequency $\left(p_{(0)}\right)$. $F_{L \& H}$ : blue line, $F_{V R}$ : brown line, FYAN: red line

is -1 (Fig. 2a). Another way of looking at this is that fixing the major allele leads to negative values for $E\left(F_{V R}\right)$ when $p_{(0)}>2 / 3$, and its limit is also -1 (Fig. 2c). In these scenarios, the value of $E\left(F_{Y A N}\right)$ remains equal to 1 . It is interesting to note that when $p_{(t)}=0.5, E\left(F_{Y A N}\right)$, and to a lesser extent $E\left(F_{V R}\right)$, behave as a mirror image of $F_{L \& H}$ (Fig. 2b).

In summary, expected values for $F_{L \& H}, F_{V R 1}, F_{V R 2}$ and $F_{Y A N}$ depend on frequency changes. When the inbreeding coefficient is interpreted as an indicator of loss or gain of variability, $F_{L \& H}$ gives sensible values but $F_{V R 1}$, $F_{V R 2}$, and $F_{Y A N}$ do not. In fact, $E\left(F_{L \& H}\right)$ follows the trend of loss or gain in heterozygosity due to changes in allele frequencies. When the minor allele frequency (MAF) decreases (i.e. when heterozygosity decreases relative to that in a reference base population), $E\left(F_{L \& H}\right)$ increases. However, $E\left(F_{V R 1}\right)$ and $E\left(F_{V R 2}\right)$ can lead us to think that: (i) more than $100 \%$ of the initial variability is lost; and, even worse, (ii) variability has increased when in reality it has decreased or vice versa. $E\left(F_{Y A N}\right)$ also leads to inconsistent results since it never indicates that variability has increased, but it can indicate that more than $100 \%$ of the initial variability is lost.

\section{Patterns of genomic inbreeding in the population of Guadyerbas pigs}

Summary statistics for the different inbreeding coefficients, computed both at the individual level and at the regional (window) level, are in Table 3 for the first and last cohorts. Average values for each coefficient at the individual and regional levels were practically the same but those at the regional level varied much more than those at the individual level, particularly for cohort 6 . The proportion of homozygous loci $\left(F_{N E J}\right)$ increased by $5 \%$ from cohort 1 to cohort 6 . Coefficient $F_{N E J}$ had a much higher average and a lower standard deviation than the other coefficients. Coefficients that are weighted by the initial frequencies (i.e. $F_{L \& H}, F_{V R 1}, F_{V R 2}$ and $F_{Y A N}$ ) were on average less than 0 for cohort 1 (about - 0.1) and became positive (up to $\sim 0.2$ ) for cohort 6 .

Pairwise correlations between coefficients computed both at the individual and regional (window) level are in Fig. 3. Correlations at the individual animal level (which are averages across the genome) ranged from 0.4 to 1 for cohort 1 and from 0.7 to 1 for cohort 6 . As expected, the correlation between $F_{N E J}$ and $F_{L \& H}$ was 1 . Correlations higher than 0.9 were also found between $F_{Y A N}$ and $F_{N E J}, F_{Y A N}$ and $F_{L \& H}, F_{Y A N}$ and $F_{V R 1}$, and $F_{V R 1}$ and $F_{V R 2}$. The lowest correlations were between $F_{V R 2}$ and $F_{N E J}$ and between $F_{V R 2}$ and $F_{L \& H}$, but these correlations increased from $\sim 0.4$ for cohort 1 to $\sim 0.7$ for cohort 6 , which could be due to the loss of rare alleles over time but also to random fluctuations. At the regional genomic level, changes in frequencies can be more exaggerated, which results in lower correlations between coefficients than at the individual animal level, particularly for those involving VanRaden's coefficients. 
Table 3 Mean, standard deviation (SD) and minimum and maximum values for the different genomic inbreeding coefficients when computed at the individual animal or genomic region level in cohorts 1 and 6 of the Guadyerbas population

\begin{tabular}{|c|c|c|c|c|c|c|c|c|c|}
\hline \multirow[t]{2}{*}{ Cohort } & & \multicolumn{4}{|c|}{ Individual level } & \multicolumn{4}{|c|}{ Regional level } \\
\hline & & Mean & SD & Min & $\operatorname{Max}$ & Mean & SD & Min & Max \\
\hline \multirow[t]{5}{*}{1} & $F_{N E J}$ & 0.616 & 0.024 & 0.580 & 0.674 & 0.615 & 0.084 & 0.300 & 0.968 \\
\hline & $F_{L \& H}$ & -0.095 & 0.070 & -0.198 & 0.071 & -0.095 & 0.122 & -0.517 & 0.373 \\
\hline & $F_{V R 1}$ & -0.095 & 0.076 & -0.230 & 0.119 & -0.095 & 0.122 & -0.517 & 0.372 \\
\hline & $F_{V R 2}$ & -0.088 & 0.108 & -0.279 & 0.211 & -0.088 & 0.106 & -0.450 & 0.340 \\
\hline & $F_{Y A N}$ & -0.088 & 0.053 & -0.165 & 0.061 & -0.088 & 0.106 & -0.450 & 0.341 \\
\hline \multirow[t]{5}{*}{6} & $F_{N E J}$ & 0.669 & 0.025 & 0.631 & 0.743 & 0.669 & 0.124 & 0.307 & 1.000 \\
\hline & $F_{L \& H}$ & 0.056 & 0.070 & -0.052 & 0.268 & 0.056 & 0.353 & -2.939 & 1.000 \\
\hline & $F_{V R 1}$ & 0.120 & 0.079 & -0.014 & 0.417 & 0.111 & 0.352 & -0.853 & 2.717 \\
\hline & $F_{V R 2}$ & 0.175 & 0.108 & 0.023 & 0.609 & 0.172 & 0.558 & -0.876 & 4.118 \\
\hline & FYAN & 0.090 & 0.076 & -0.014 & 0.364 & 0.089 & 0.215 & -0.465 & 1.306 \\
\hline
\end{tabular}

The pattern of homozygosity clearly varied across chromosomes and across regions within chromosomes (Fig. 4). For several genomic regions, SNPs that were still segregating in cohort 1 became fixed in cohort 6 (see for example, Sus scrofa (SSC) chromosomes 4, 8, 13, 14 and 17).

Figure 5 compares the patterns of the different coefficients across the genome for cohort 6 . Here, we only consider the SNPs that segregated in cohort 1 . In general, the patterns differed a lot between coefficients. It is interesting to note that, in general, the patterns for $F_{V R 1}$ and $F_{V R 2}$ were mirror images of those for $F_{L \& H}$. One particularly striking result is that in regions where SNPs had become fixed (see also Fig. 4), $F_{L \& H}$ was equal to 1 whereas $F_{V R 1}$ and $F_{V R 2}$ were negative with large absolute values. Two very clear examples are the region between 43 and $56 \mathrm{Mb}$ on SSC4 and the region between 58 and $82 \mathrm{Mb}$ on SSC14. In both these regions, the initial frequency of the minor allele was very low $\left(p_{(0)} \leq 0.1\right)$, and the allele was already lost in cohort $6\left(p_{(t)}=0\right)$. At all positions within these regions, $F_{L \& H}$ was equal to 1 , while $F_{V R 1}$ and $F_{V R 2}$ became negative (about -0.8 in the SSC4 region and ranging from -0.9 to -0.6 in the $\mathrm{SSC} 14$ region), which incorrectly suggests that some variability was gained, and $F_{Y A N}$ was low (about 0.1 in the SSC4 region and ranging from 0.1 to 0.2 in the SSC14 region). These observations agree with the expectations described above and lead us to conclude that $F_{L \& H}$ is a much more valuable measure of change in variability than $F_{V R 1}$ or $F_{V R 2}$. For the regions where all variability was lost, $F_{L \& H}$ is expected to indicate that this is the case, but both $F_{V R 1}$ and $F_{V R 2}$ indicate that variability was gained.

In addition, there are some regions for which the variability increased from cohort 1 to cohort 6, as $F_{N E J}$ was lower in the latter (Fig. 4), e.g. the regions between 102 and $112 \mathrm{Mb}$ on SSC3, between 41 and $72 \mathrm{Mb}$ on SSC6, and between 81 and $97 \mathrm{Mb}$ on SSC13. In all these cases, $F_{L \& H}$ did indeed show this increase in variability since it became negative. However, $F_{V R 1}$ and $F_{V R 2}$ were again like mirror images of $F_{L \& H}$, while $F_{Y A N}$ was positive but close to 0 . These observations also agree with expectations. For instance, the average $p_{(0)}$ and $p_{(t)}$ in the SSC13 region were 0.31 and 0.40 , respectively. With this change in frequency, $E\left(F_{L \& H}\right)$ varied from -1 to 0 , while the expected values for $F_{V R 1}, F_{V R 2}$ and $F_{Y A N}$ were all between 0 and 1. Also remarkable are the high peaks observed for $F_{V R 2}$. In some regions on SSC2 and SSC17, $F_{V R 2}$ reached a value as high as 4 . In these regions (between 35 and $38 \mathrm{Mb}$ on SSC2 and between 33 and $34 \mathrm{Mb}$ on SSC17), there are SNPs with rare alleles $\left(p_{(0)}<0.1\right)$ which had a high increase in frequency $\left(p_{(t)}>0.3\right)$, and under these circumstances, $F_{V R 2}$ is expected to reach very high positive values (Fig. 1b), while $F_{L \& H}$ is expected to become negative (Fig. 1a).

With VanRaden's and Yang's coefficients, and in particular $F_{V R 2}$, a higher inbreeding coefficient is assigned to an individual that is homozygous for a rare allele than to an individual that is homozygous for a common allele. Thus, $F_{V R 1}, F_{V R 2}$, and $F_{Y A N}$ put a greater weight on SNPs that have a low MAF. Based on this, in addition to the scenario considered so far, in which all the SNPs segregating in cohort $1(\mathrm{MAF}>0)$ were used to calculate the inbreeding coefficients, we analyzed two additional scenarios with different MAF thresholds in cohort 1: (i) using only the common variants (here defined as SNPs with MAF >0.05); and (ii) using only the very common variants (here defined as SNPs with MAF $>0.25$ ). This allowed us to determine how the differences between coefficients were affected by MAF. 

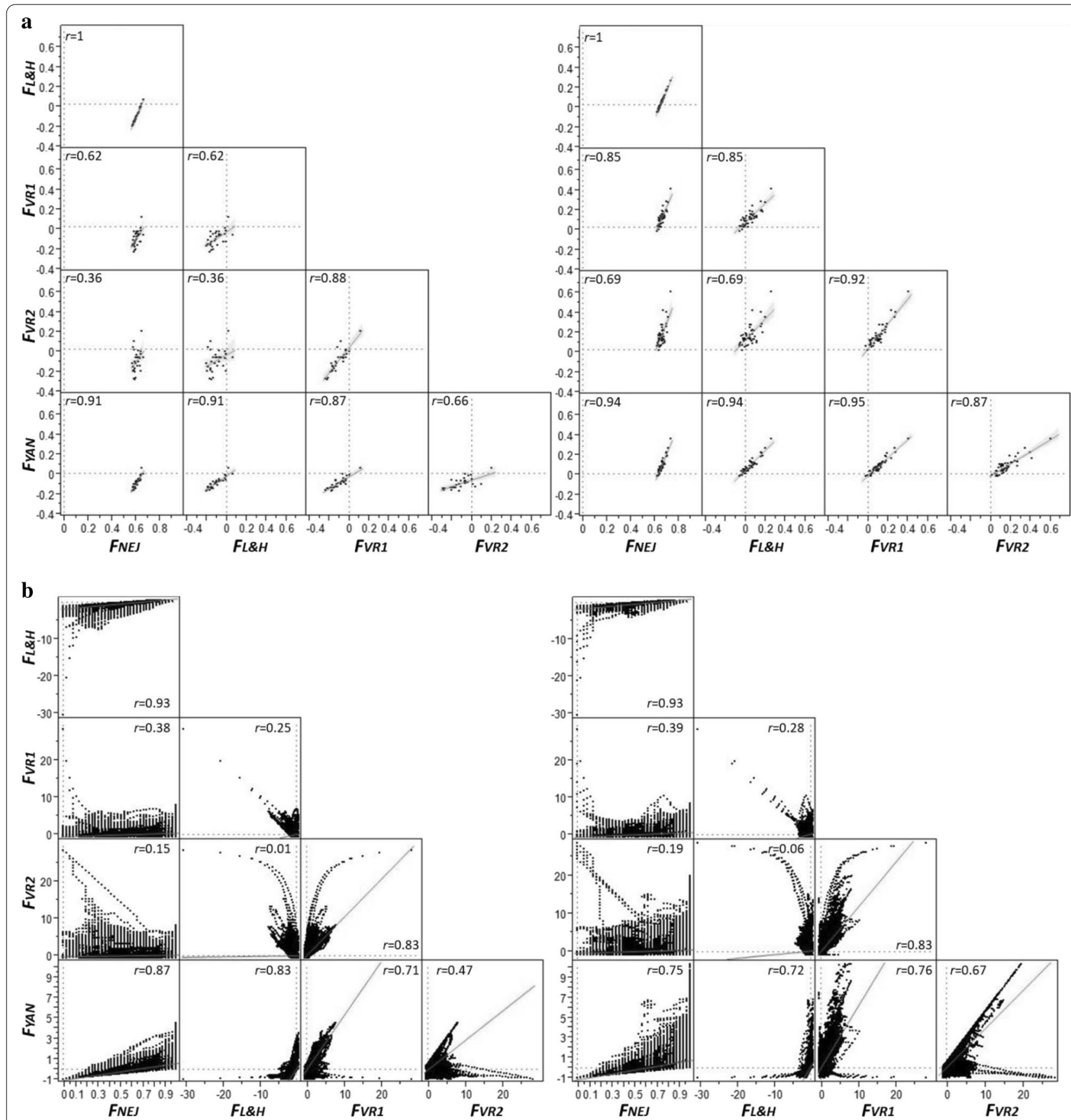

Fig. 3 Scatter plots for inbreeding coefficients $F_{N E J}, F_{L \& H}, F_{V R 1}, F_{V R 2}$ and $F_{Y A N}$ in the Guadyerbas population when computed at the individual animal (a) or genomic region (b) level in cohorts 1 (left panels) and 6 (right panels), and the corresponding correlation coefficients $(r)$

Figure 6 shows the patterns of each coefficient computed using only SNPs with a MAF $>0.05$ or $>0.25$ for three chromosomes. When only SNPs with a MAF higher than 0.05 in cohort 1 were used, some of the strong peaks previously obtained disappeared, in particular for $F_{V R 2}$ (Fig. 6 versus Fig. 5). Using an even stricter MAF filter $(\mathrm{MAF}>0.25)$ led to very similar patterns for all inbreeding coefficients (Fig. 6). In fact, pairwise correlations between coefficients increased considerably compared to those shown in Fig. 3. When only SNPs with a MAF higher than 0.25 were used, all correlations were higher than 0.95 , both in cohorts 1 and 6. SNPs with a MAF higher than 0.05 and higher than 0.25 represented $92 \%(16,532$ SNPs) and 54\% 

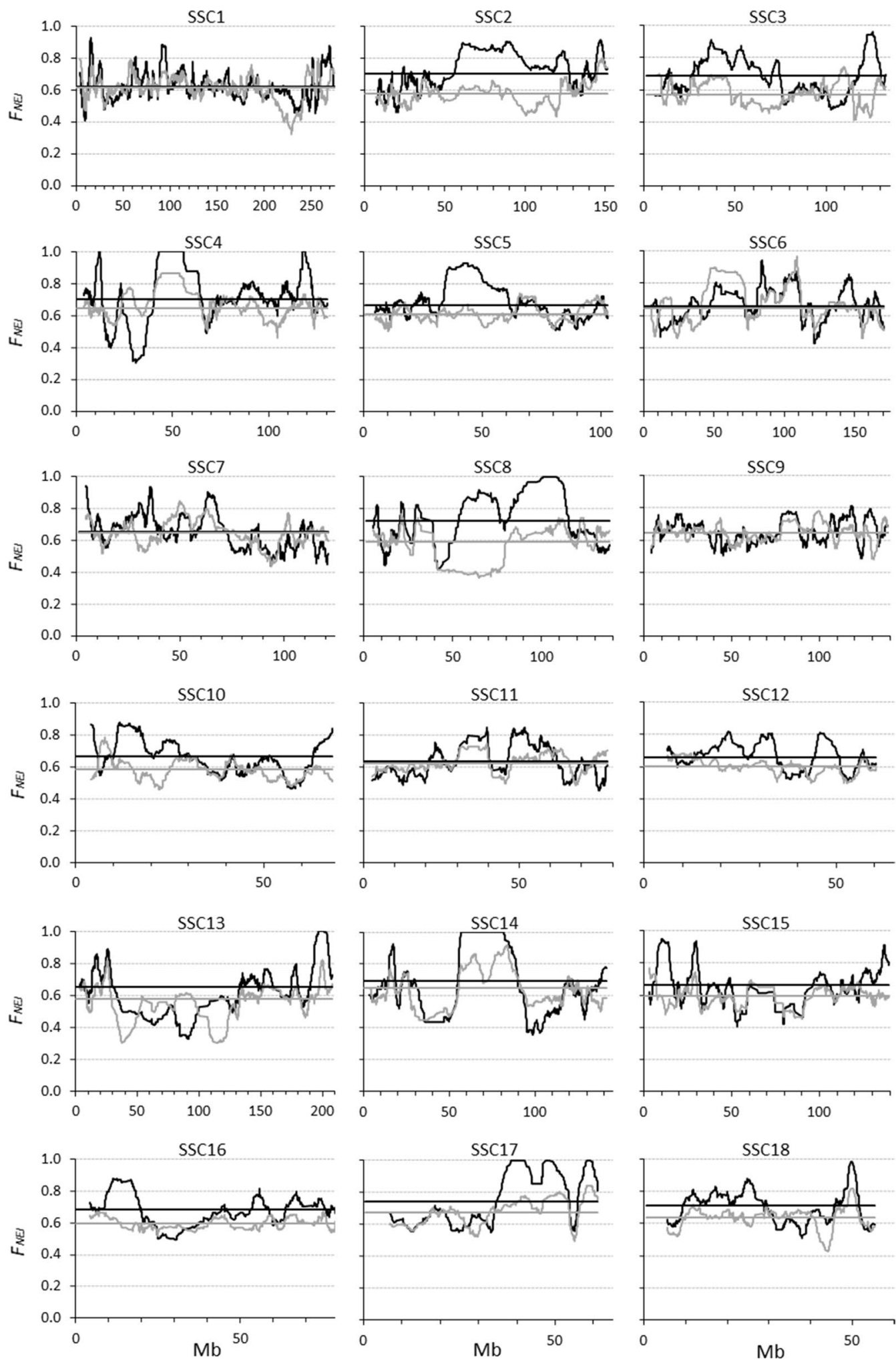

Fig. 4 Evolution of the proportion of the genome that becomes homozygous (i.e. $F_{\text {NE) }}$ ) from cohort 1 (grey lines) to cohort 6 (black lines) for the different chromosomes (SSC) in the Guadyerbas population when using SNPs with non-zero minor allele frequencies. The horizontal lines represent averages across the genome 


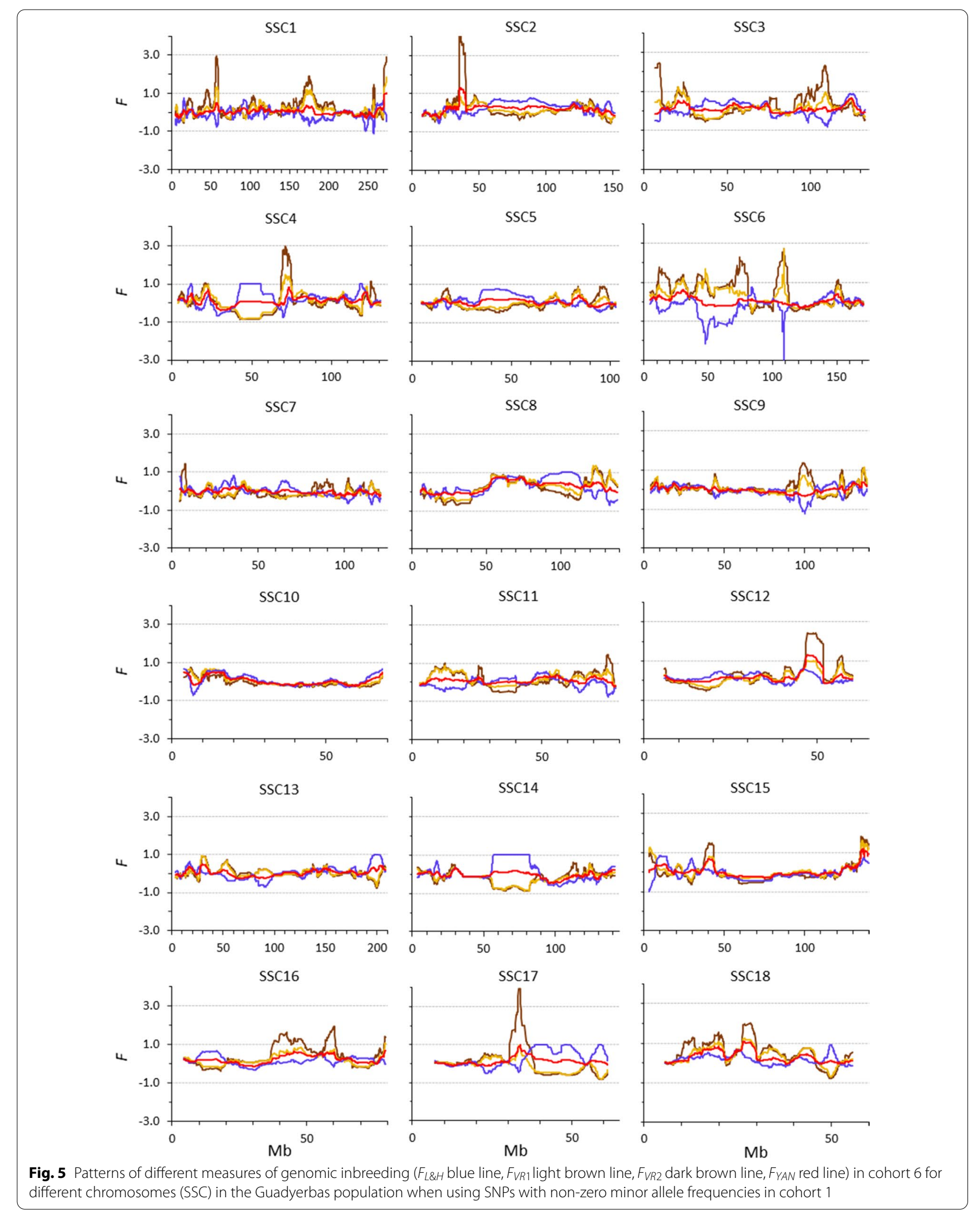



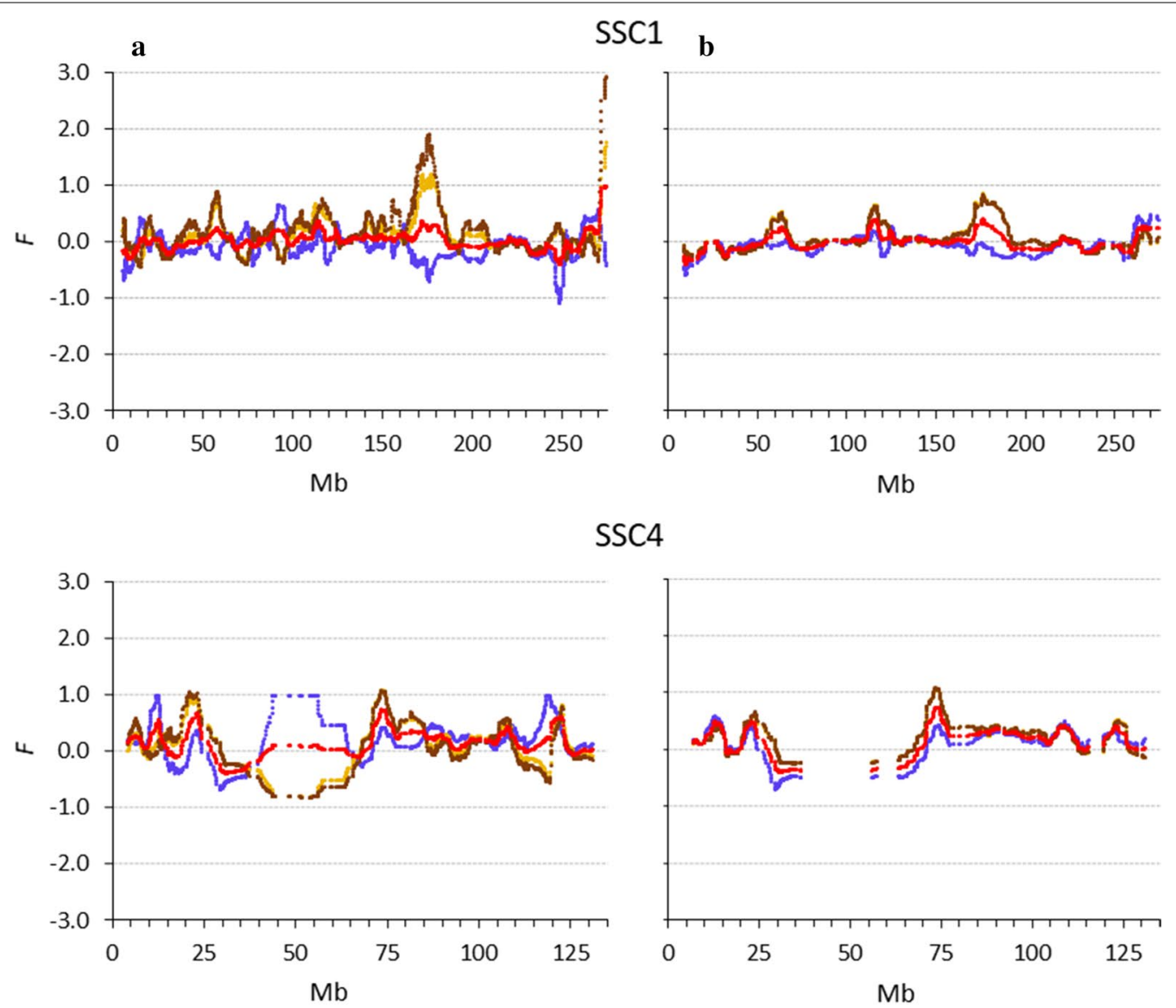

SSC17
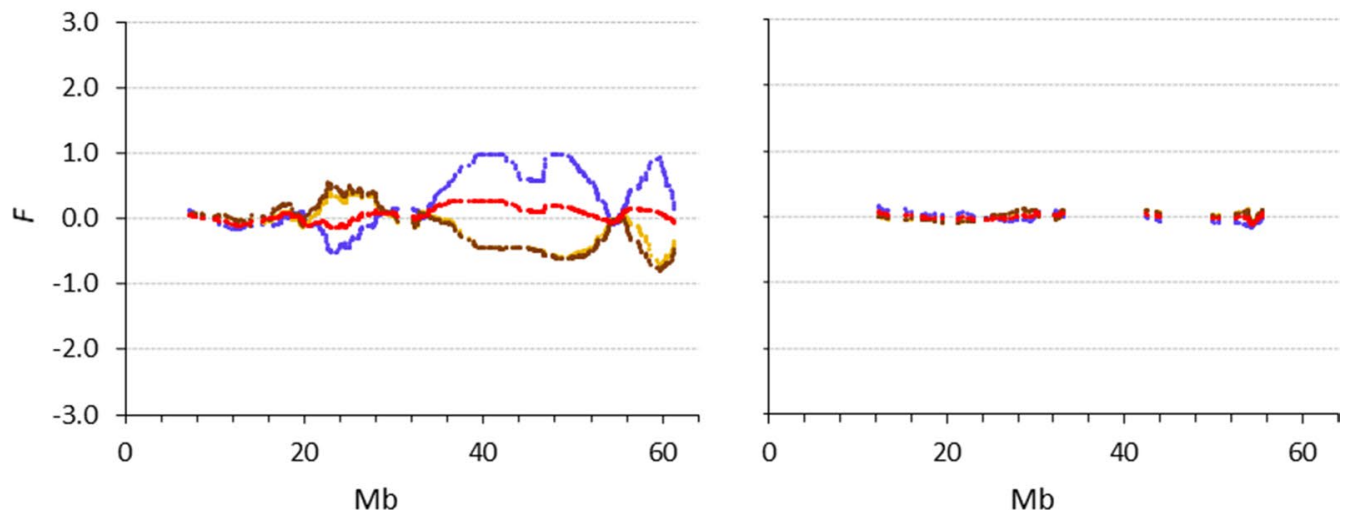

Fig. 6 Patterns of different measures of genomic inbreeding ( $F_{L \& H}$ blue line, $F_{V R 1}$ light brown line, $F_{V R 2}$ dark brown line, $F_{Y A N}$ red line) in cohort 6 for chromosomes 1, 4, and 17 in the Guadyerbas population when using SNPs with minor allele frequencies $>0.05$ (a) or $>0 ; 25$ (b) in cohort 1

(9,716 SNPs), respectively, of the total number of segregating SNPs in cohort 1 . Note that SNP density greatly decreased in some regions when SNPs were filtered on MAF, resulting in the discontinuities seen in Fig. 6 . These results show that the inconsistencies described earlier for $F_{V R 1}, F_{V R 2}$, and $F_{Y A N}$ occur when there are SNPs with a low MAF, and in practice such SNPs exist. Removing loci with rare alleles would defeat the rationale behind the coefficients that intentionally give more weight to rare alleles. 


\section{Consequences for the estimation of inbreeding depression}

For each of the three measures of $F$, the patterns of the rate of inbreeding depression (i.e. the regression coefficient, $b$ ) for all chromosomes are shown in Additional file 2: Figure S2. Across the whole genome, the estimates of $b$ differed substantially between the methods used to compute $F$. In some regions within chromosomes, estimates of $b$ were very similar across methods but in other regions they differed greatly, not only in magnitude but also in sign. As an illustration, Fig. 7 shows selected regions within chromosomes for which the conclusions on the magnitude and sign of the rate of inbreeding depression differ substantially. In the regions from 50 to $70 \mathrm{Mb}$ and from 98 and $109 \mathrm{Mb}$ on SSC6, estimates of $b$ were close to 0 when using $F_{L \& H}$ and $F_{V R 2}$ but clearly different from 0 when using $F_{Y A N}$. However, in other regions (e.g. from 90 to $113 \mathrm{Mb}$ on SSC8, from 20 to $24 \mathrm{Mb}$ on SSC10, from 50 to $65 \mathrm{Mb}$ on SSC14, and from 56 to $60 \mathrm{Mb}$ on SSC17), $F_{L \& H}$ and $F_{Y A N}$ led to estimates of $b$ that were of the same sign but opposite to estimates obtained when using $F_{V R 2}$. In the region from 7.5 to $11 \mathrm{Mb}$ on SSC18, the sign of the estimate of $b$ obtained with $F_{L \& H}$ was opposite to that obtained with $F_{V R 2}$ and $F_{Y A N}$.

Pairwise correlations between estimates of rates of inbreeding depression computed with $F_{L \& H}, F_{V R 2}$, and $F_{Y A N}$ are in Additional file 3: Figure S3. Across the genome, correlations involving estimates based on $F_{V R 2}$ ranged from $\sim 0.4$ to 0.5 , whereas the correlation between estimates based on $F_{L \& H}$ and $F_{Y A N}$ was high (0.84). About $40 \%$ of the estimates of $b$ in Additional file 3: Figure S3 were of opposite sign when based on $F_{L \& H}$ and $F_{V R 2}$, and this percentage decreased to $27 \%$ when estimates were based on $F_{V R 2}$ and $F_{Y A N}$ and to $15 \%$ when using $F_{L \& H}$ and $F_{Y A N}$. This reinforces the idea that care should be taken when interpreting estimates of inbreeding depression obtained with different measures of genomic inbreeding.

\section{Discussion}

The inbreeding coefficient has been defined as a probability [9] or as a correlation [8], and thus its legitimate range is between 0 and 1 or between -1 and 1 , respectively. Another interpretation of the inbreeding coefficient, which we have used here, is in terms of loss or gain of variability relative to a reference base population. Under this interpretation, on the one hand, a negative value (even a value lower than -1 ) makes sense and means that some variability has been gained. On the other hand, a value higher than 1 means that more variability than that initially existing has been lost, which is not reasonable.

Using a single locus model, we provided expectations for different genomic inbreeding coefficients that have been widely used in the literature. These expectations help to understand the patterns of these coefficients when they are computed using thousands of SNPs in a real population. Except for $F_{N E J}$, none of the genomic coefficients considered here (i.e. those depending on allele frequencies) match with Malécot's or Wright's definition of the inbreeding coefficient as a probability or correlation, respectively, since their values can be outside
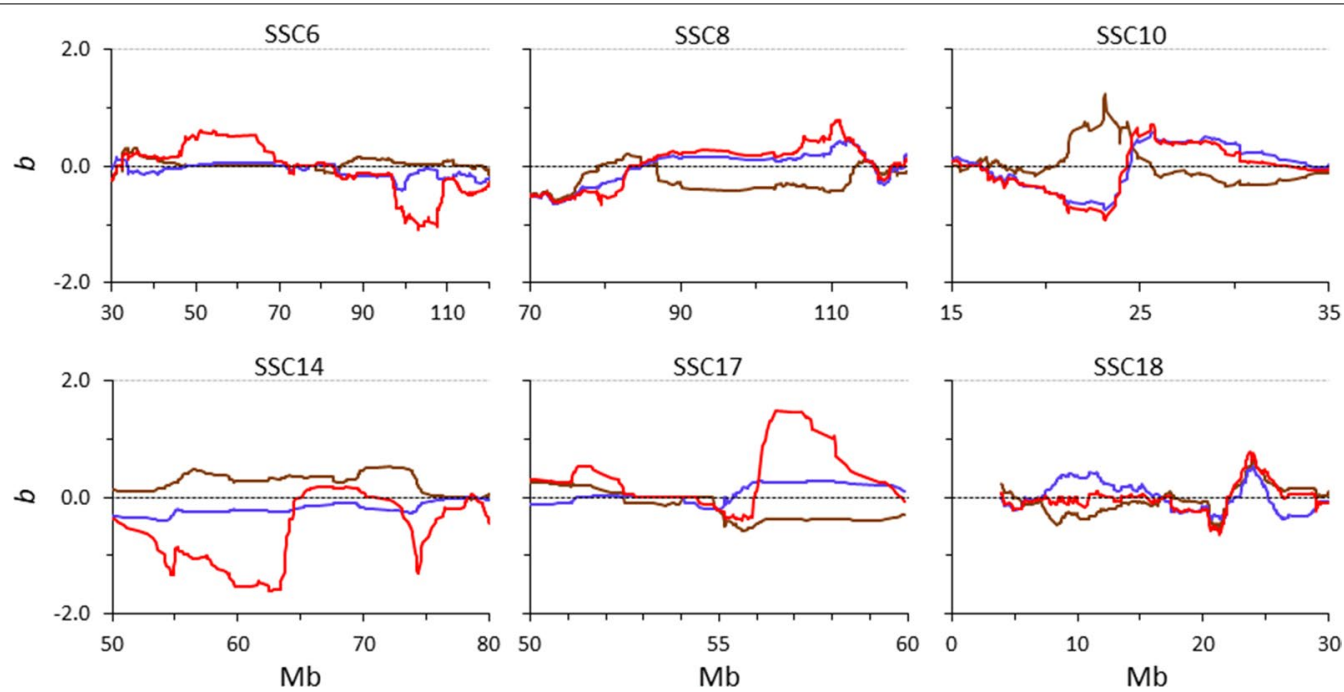

Fig. 7 Patterns of the rate of inbreeding depression (b) for number of piglets born alive in the Guadyerbas population when computed using different measures of genomic inbreeding ( $F_{L \& H}$ blue line, $F_{V R 2}$ brown line, $F_{Y A N}$ red line) for specific regions of six chromosomes. All genotyped sows with phenotypic data that were born from cohort 1 to cohort 6 were included in the analyses 
the legitimate ranges [47]. In fact, at the individual animal level, $F_{L \& H}$ can range from $-\infty$ to 1 and $F_{V R 1}, F_{V R 2}$, and $F_{Y A N}$ from -1 to $\infty$. At the population level (see the section on expected genomic coefficients under a single locus model above), the ranges are the same as at the individual level, except for $F_{Y A N}$, which can range from 0 to $\infty$. When these coefficients are interpreted as indicators of whether variability is gained or lost over generations, $F_{L \& H}$ leads to sensible results but $F_{V R 1}, F_{V R 2}$, and $F_{Y A N}$ do not. This also has consequences when estimating the rate of inbreeding depression for specific genome regions since different measures of inbreeding can lead to very different results.

Although $F_{L \& H}$ is not a probability or a correlation, this measure of inbreeding is useful for determining whether variability is lost or gained. The largest variability (heterozygosity) for biallelic loci occurs at allele frequencies equal to 0.5 . When a rare allele increases its frequency towards $0.5, F_{L \& H}$ indicates that variability is gained, as expected. In addition, this measure of inbreeding never indicates that more variability than what existed in the initial generation was lost. In contrast, $F_{V R 1}, F_{V R 2}$, and $F_{L \& H}$ also do not match with a definition of inbreeding based on the proportion of variability lost or gained. In fact, for some $p_{(0)}$ and $p_{(t)}$ combinations, these three coefficients can indicate that variability is lost when the MAF increased towards 0.5 , and this loss can be even higher than $100 \%$ of the initial variability. Moreover, $F_{V R 1}$ and $F_{V R 2}$ can take values that indicate that heterozygosity in the current generation is higher than what existed in the initial generation, although some heterozygosity has actually been lost. This does not occur with $F_{L \& H}$ at the population level since $E\left(F_{L \& H}\right)$ is never negative, i.e. it always indicates that heterozygosity decreases although, in theory, it could increase.

One of the advantages of using genomic rather than pedigree data to measure inbreeding is the possibility of investigating the pattern of inbreeding along the genome. Here, we compared the patterns of each genomic coefficient computed from thousands of SNPs obtained with the Illumina PorcineSNP60 BeadChip v1 in a population of Iberian pigs. This population is highly inbred, with an estimated effective population size as low as $20[54,55]$. The behaviour of each inbreeding coefficient observed with real data was well explained by the expectations developed. In the six generations (i.e. from cohort 1 to cohort 6), many SNPs became fixed (see Fig. 4). This loss of variability was captured by $F_{N E J}$ (which is simply the proportion of homozygous SNPs) and was also clearly reflected in the value for $F_{L \& H}\left(F_{L \& H}=1\right)$. However, the negative values obtained for $F_{V R 1}$ and $F_{V R 2}$ for these regions indicate that variability in cohort 6 was higher than in cohort 1 . For regions where the variability increased from cohort 1 to cohort $6, F_{L \& H}$ had negative values (which reflects reality), while $F_{V R 1}, F_{V R 2}$, and $F_{Y A N}$ had positive values (which do not reflect the reality). Although $E\left(F_{Y A N}\right)$ predicts that variability can be gained in some circumstances, this occurs when, in fact, variability has been lost. In general, $F_{V R 1}$ and $F_{V R 2}$ behave similarly, although values for $F_{V R 2}$ are more extreme. Values for $F_{Y A N}$ lie between those for $F_{L \& H}$ and those for $F_{V R 1}$ and $F_{V R 2}$, and generally, are close to 0 . Given these results, it is clear that, when specific genome regions are targeted to control inbreeding, the choice of the genomic coefficient used should be done with care.

We have analysed the behaviour of five genomic measures of inbreeding that have been widely used in the literature. However, other measures have been proposed (see review by Kardos et al. [32]). For instance, both the PLINK [56] and GCTA [18] software provide a modification of $F_{L \& H}$ (their $F^{I I}$, here referred to as $F_{L \& H 2}$ ). Although to our knowledge, $F_{L \& H 2}$ is not widely used, it is interesting to note that the difference between $F_{L \& H}$ and $F_{L \& H 2}$ is equivalent to the difference between $F_{V R 1}$ and $F_{V R 2}$ in that it only differs in how the summation over SNPs is carried out. This is clearly illustrated by the patterns of these coefficients obtained for the Guadyerbas pig population (see Additional file 4: Figure S4). The patterns for $F_{L \& H}$ were, in general, mirror images of the patterns for $F_{V R 1}$ and those for $F_{L \& H 2}$ were, in general, mirror images of patterns for $F_{V R 2}$. Another widely used measure of genomic inbreeding, which we have not considered here, is the coefficient $F_{R O H}$ based on continuous runs of homozygosity $(\mathrm{ROH})[11,12]$. Contrary to the coefficients considered here, which are computed on a SNP-by-SNP basis, $F_{R O H}$ is computed on a segment basis and has the advantages that (i) its values range from 0 to 1 ( $F_{R O H}$ is the proportion of the genome that is in $\mathrm{ROH}$ ); and (ii) it can distinguish between distant (based on short $\mathrm{ROH}$ ) and recent (based on long $\mathrm{ROH}$ ) inbreeding. Its ability to detect inbreeding depression has been proven in multiple studies $[3,11,12,19,21,25,26,28,32$, $36,39,40,42,57]$. However, the exact definition of $F_{R O H}$ varies across studies, depending on the choice of the parameters to define a $\mathrm{ROH}$ (e.g. number of heterozygous genotypes permitted in a $\mathrm{ROH}$, minimum SNP density required, maximum distance allowed between two consecutive homozygous SNPs, and minimum number of SNPs). Because of this, population-wide expected values for $F_{R O H}$ are difficult to derive.

The pedigree-based numerator relationship matrix, NRM [58] has been used very extensively for many years to estimate the genetic covariance between individuals that are genetically evaluated via best linear unbiased prediction (BLUP). With the advent of genomic evaluations [59], the NRM has been replaced by more 
precise realised relationship matrices, which has led to an increase in the accuracy of predicted breeding values (e.g. [60]). Replacing NRM with GRM has also led to two other applications. The first application was the object of our study. Given that self-relationships in the NRM are expected to be equal to 1 plus the individual's inbreeding coefficient, genomic inbreeding coefficients have been also obtained from the diagonals of the GRM. However, as we have shown here, this is not always justified. In the ideal situation, with an infinite number of independent loci and absence of migration, mutation, and selection, the average allele frequencies remain constant over generations and all measures, except $F_{N E J}$, are expected to produce unbiased estimates of the inbreeding coefficient (IBD) relative to a base population that is in Hardy-Weinberg equilibrium [42, 61]. However, in more realistic situations, the proposed genomic estimators of inbreeding can result in very different outcomes. The second application of the GRM is based on the fact that the NRM is equal to twice the matrix of coancestry coefficients and, as such, it has been used to optimize contributions of breeding candidates by applying the optimal contribution method (OC) for maintaining genetic variability and avoiding inbreeding depression in genetic conservation programs $[47,62,63]$. In this context, GRM have been used in OC, replacing NRM. de Cara et al. [64] and Gomez-Romano et al. [65] showed that the use of the coancestry matrix computed from Nejati-Javaremi's GRM in OC resulted in a higher level of genetic diversity (measured as expected heterozygosity) being maintained than when using the NRM in OC. Morales-González et al. [47] showed that the amount of genetic variability retained was higher when using Nejati-Javaremi's or Li and Horvitz's matrices in OC than when using VanRaden and Yang's GRM, although the latter were also efficient in controlling the loss of genetic diversity. Thus, in the context of optimizing contributions for maintaining diversity, VanRaden and Yang's GRM are useful. In fact, it has been suggested [66] that although the use of VanRaden and Yang's GRM in OC results in less variability being maintained, they could lead to allele frequencies that are closer to those in the original population (i.e. allele frequencies would tend to be unchanged), which can be an objective in conservation programs, particularly in ex situ conservation programs, where the final aim is reintroduction to the wild [67].

It has been suggested that the use of whole-genome sequence data could produce improved genomic inbreeding coefficient estimates [23] because it captures the many variants with rare alleles, which may not be included in the SNP panels due to their ascertainment bias. However, including a higher proportion of variants with rare alleles is expected to lead to even more inconsistent results than those shown here when using $F_{Y A N}$ and $F_{V R 1}$, and particularly $F_{V R 2}$.

Under the infinitesimal model, the NRM is a matrix of covariances of breeding values but, importantly, it is also twice the matrix of coancestry coefficients, with selfcoancestries on the diagonal. Given that the relationship between self-coancestry $(f)$ and inbreeding $(F)$ coefficients is $f=0.5(1+F)$, the NRM provides estimates of $F$. GRM are also covariance matrices that have proven to work very well in genomic predictions. However, although $F_{N E J}$ and $F_{L \& H}$ correctly indicate when variability is lost or gained, this is not the case with $F_{V R 1}, F_{Y V R 2}$, and $F_{Y A N}$.

\section{Conclusions}

Except for $F_{N E J}$ (which ranges from 0 to 1 ), values for the genomic coefficients investigated here are outside the ranges of Malécot's and Wright's definitions of coefficient of inbreeding. When using a third interpretation of inbreeding in terms of loss or gain of variability, $F_{L \& H}$ gives sensible values but $F_{V R 1}, F_{V R 2}$, and $F_{Y A N}$ do not. In fact, the expectations derived here at the population level show some inconsistencies for these three coefficients. These include indications that (i) more variability than what initially existed can be lost $\left(F_{V R 1}, F_{V R 2}\right.$, and $\left.F_{Y A N}\right)$; (ii) variability has decreased when in reality it has increased $\left(F_{V R 1}, F_{V R 2}\right.$, and $\left.F_{Y A N}\right)$; (iii) variability has increased when in reality it has decreased $\left(F_{V R 1}\right.$ and $\left.F_{V R 2}\right)$; and (iv) it is not possible to gain more variability than what existed initially $\left(F_{Y A N}\right)$. The expectations developed here clearly explain the different patterns of these coefficients obtained for a highly inbred pig population when using thousands of SNP genotypes.

\section{Supplementary Information}

The online version contains supplementary material available at https://doi. org/10.1186/s12711-021-00635-0.

Additional file 1: Figure S1. Expected $F_{L \& H}, F_{V R}\left(F_{V R 1}=F_{V R 2}\right)$ and $F_{Y A N}$ at a given current frequency $\left(p_{(t)}\right)$ when the initial frequency of the reference allele $\left(p_{(0)}\right)$ is set to 0.5 . $F_{L \& H}$ : blue line, $F_{V R}$ : brown line, and $F_{Y A N}$ : red line.

Additional file 2: Figure S2. Patterns of the rate of inbreeding depression for number of piglets born alive $(\boldsymbol{b})$ when computed using different measures of genomic inbreeding $\left(F_{L \& H}\right.$ : blue line, $F_{V R 2}$ : brown line, and $F_{Y A N}$ : red line) for each chromosome of the Guadyerbas genome. All genotyped sows with phenotypic data that were born from cohort 1 to cohort 6 were included in the analyses.

Additional file 3: Figure $\mathbf{S 3}$. Scatter plots for rates of inbreeding depression for number of piglets born alive $(\boldsymbol{b})$ when computed using $F_{L \& H}$, $F_{V R 2}$ or $F_{Y A N}$ against each other, and corresponding correlation coefficients ( $r$. All genotyped sows with phenotypic data that were born from cohort 1 to cohort 6 were included in the analyses. Values indicated with different colors correspond to regions presented in Fig. 7.

Additional file 4: Figure S4. Patterns of different measures of genomic inbreeding $\left(F_{L \& H}\right.$ : blue line, $F_{L \& H 2}$ : grey line, $F_{V R 1}$ : light brown line, 
$F_{V R 2}$ : dark brown line, and $F_{Y A N}$ : red line) in cohort 6 for each chromosome of the Guadyerbas genome when using SNPs with a MAF higher than 0 in cohort 1 .

\section{Acknowledgements}

We are grateful to Jaime Rodrigáñez, Luis Silió and the staff of the CIA 'Dehesón del Encinar' (Oropesa, Toledo) for their excellent job in maintaining the Guadyerbas herd for decades. We thank the 'Centro de Supercomputación de Galicia' (Cesga) for their computing support and two anonymous referees for helpful comments on the manuscript.

\section{Authors' contributions}

$\mathrm{BV}$ and RPW conceived and designed the study. AF performed the analyses and constructed the figures. BV wrote the first draft of the manuscript. All authors contributed to the discussion of results and the edition of the manuscript revision. All authors read and approved the final manuscript.

\section{Funding}

This work was carried out with funding from the Ministerio de Ciencia e Innovación, Spain (CGL2016-75904-C2, PID2020-114426GB-C22), the European Commission Horizon 2020 (H2020) Framework Programme through grant agreement no 727315 MedAID project (Mediterranean Aquaculture Integrated Development), Xunta de Galicia (ED431C 2020-05) and Fondos FEDER. R. Pong-Wong is funded by the European Union's Horizon 2020 research and innovation program under the Grant Agreement $n^{\circ} 772787$ (SMARTER) and the Biotechnology and Biological Sciences Research Council through Institute Strategic Programme Grant funding (BBS/E/D/30002275).

\section{Availability of data and materials}

The datasets analyzed during the current study are available from the corresponding author on reasonable request.

\section{Declarations}

\section{Ethics approval and consent to participate}

Ethic Committee approval was not obtained for this study because no new animals were handled in this experiment. Analyses were performed using data previously collected with approval by the INIA Scientific Ethic Committee. Animal manipulations were performed according to the Spanish Policy for Animal Protection RD1201/05, which meets the European Union Directive 86/609 about the protection of animals used in experimentation.

\section{Consent for publication}

Not applicable.

\section{Competing interests}

The authors declare that they have no competing interests.

\section{Author details}

'Departamento de Mejora Genética Animal, INIA, Ctra. de La Coruña, km 7.5, 28040 Madrid, Spain. ${ }^{2}$ Centro de Investigación Mariña, Universidade de Vigo, Departamento de Bioquímica, Genética E Inmunología, Campus de Vigo, 36310 Vigo, Spain. ${ }^{3}$ Departamento de Producción Agraria, ETSI Agrónomos, Universidad Politécnica de Madrid, 28040 Madrid, Spain. ${ }^{4}$ Genetics and Genomics, The Roslin Institute and the R(D)SVS, University of Edinburgh, Easter Bush, Midlothian EH25 9RG, UK.

\section{Received: 17 July 2020 Accepted: 19 April 2021}

Published online: 01 May 2021

\section{References}

1. Walsh B, Lynch M. Evolution and selection of quantitative traits. Oxford: Oxford University Press; 2018

2. McQuillan R, Eklund N, Pirastu N, Kuningas M, McEvoy B, Esko T, et al. Evidence of inbreeding depression in human height. PLoS Genet. 2012;8:e1002655.
3. Yengo L, Wray NR, Visscher PM. Extreme inbreeding in a European ancestry sample from the contemporary UK population. Nat Commun. 2019:10:3719.

4. Roff DA. Evolutionary quantitative genetics. New York: Chapman \& Hall; 1997.

5. Frankham R, Ballou JD, Briscoe DA. Introduction to conservation genetics. 2nd ed. Cambridge: Cambridge University Press; 2010.

6. Falconer DS, MacKay TFC. Introduction to quantitative genetics. 4th ed. Harlow: Pearson Longman; 1996.

7. Caballero A. Quantitative genetics. Cambridge: Cambridge University Press; 2020.

8. Wright S. Systems of mating. Genetics. 1921;6:111-78.

9. Malécot G. Les mathématiques de l'hérédité. Paris: Masson et Cie; 1948.

10. Wright S. Coefficients of inbreeding and relationships. Am Nat. 1922;56:330-9.

11. Keller MC, Visscher PM, Goddard ME. Quantification of inbreeding due to distant ancestors and its detection using dense single nucleotide polymorphism data. Genetics. 2011;189:237-49.

12. McQuillan R, Leutenegger AL, Abdel-Rahman R, Franklin CS, Pericic M, Barac-Lauc L, et al. Runs of homozygosity in European populations. Am J Hum Genet. 2008;83:359-72.

13. Li CC, Horvitz DG. Some methods of estimating the inbreeding coefficient. Am J Hum Genet. 1953;5:107-17.

14. Nejati-Javaremi A, Smith C, Gibson JP. Effect of total allelic relationship on accuracy of evaluation and response to selection. J Anim Sci. 1997;75:1738-45.

15. VanRaden PM. Efficient methods to compute genomic predictions. J Dairy Sci. 2008;91:4414-23.

16. Yang J, Benyamin B, McEvoy BP, Gordon S, Henders AK, Nyholt DR, et al Common SNPs explain a large proportion of the heritability for human height. Nat Genet. 2010;42:565-9.

17. VanRaden PM, Olson KM, Wiggans GR, Cole JB, Tooker ME. Genomic inbreeding and relationships among Holsteins, Jerseys, and Brown Swiss. J Dairy Sci. 2011;94:5673-82.

18. Yang J, Lee SH, Goddard ME, Visscher PM. GCTA: a tool for genome-wide complex trait analysis. Am J Hum Genet. 2011;88:76-82.

19. Bjelland DW, Weigel K, Vukasinovic N, Nkrumah JD. Evaluation of inbreeding depression in Holstein cattle using whole-genome SNP markers and alternative measures of genomic inbreeding. J Dairy Sci. 2013;96:4697-706.

20. Saura M, Fernández A, Rodríguez MC, Toro MA, Barragán C, Fernández Al, Villanueva B. Genome-wide estimates of coancestry and inbreeding in a closed herd of Iberian pigs. PLoS One. 2013;8:e78314.

21. Pryce JE, Haile-Mariam M, Goddard ME, Hayes BJ. Identification of genomic regions associated with inbreeding depression in Holstein and Jersey dairy cattle. Genet Sel Evol. 2014;46:71.

22. Wang J. Marker-based estimates of relatedness and inbreeding coefficients: an assessment of current methods. J Evol Biol. 2014;27:518-30.

23. Eynard SE, Windig JJ, Leroy G, van Binsbergen R, Calus MPL. The effect of rare alleles on estimated genomic relationships from whole genome sequence data. BMC Genet. 2015;16:24.

24. Howard JT, Haile-Mariam M, Pryce JE, Maltecca C. Investigation of regions impacting inbreeding depression and their association with the additive genetic effect for United States and Australia Jersey dairy cattle. BMC Genomics. 2015;16:813.

25. Kardos M, Luikart G, Allendorf FW. Measuring individual inbreeding in the age of genomics: marker-based measures are better than pedigrees. Heredity (Edinb). 2015;115:63-72.

26. Saura M, Fernández A, Varona L, Fernández Al, de Cara MAR, Barragán C, Villanueva B. Detecting inbreeding depression in reproductive traits in Iberian pigs using genome-wide data. Genet Sel Evol. 2015;47:1.

27. Zhang Q, Calus MPL, Guldbrandtsen B, Lund MS, Sahana G. Estimation of inbreeding using pedigree, 50k SNP chip genotypes and full sequence data in three cattle breeds. BMC Genet. 2015;16:88.

28. Bérénos C, Ellis PA, Pilkington JG, Pemberton JM. Genomic analysis reveals depression due to both individual and maternal inbreeding in a freeliving mammal population. Mol Ecol. 2016;25:3152-68.

29. Eynard SE, Windig JJ, Hiemstra SJ, Calus MPL. Whole-genome sequence data uncover loss of genetic diversity due to selection. Genet Sel Evol. 2016:48:33. 
30. Garbe JR, Prakapenka D, Tan C, Da Y. Genomic inbreeding and relatedness in wild panda populations. PLoS One. 2016;11:e160496.

31. Huisman J, Kruuk LEB, Ellis PA, Clutton-Brock T, Pemberton JM. Inbreeding depression across the lifespan in a wild mammal population. Proc Natl Acad Sci USA. 2016;113:3585-90.

32. Kardos M, Taylor HR, Ellegren H, Luikart G, Allendorf FW. Genomics advances the study of inbreeding depression in the wild. Evol Appl. 2016;9:1205-18

33. Mastrangelo S, Tolone M, Di Gerlando R, Fontanesi L, Sardina MT, Portolano B. Genomic inbreeding estimation in small populations: evaluation of runs of homozygosity in three local dairy cattle breeds. Animal. 2016;10:746-54.

34. Brito LF, Kijas JW, Ventura RV, Sargolzaei M, Porto-Neto LR, Cánovas A, et al. Genetic diversity and signatures of selection in various goat breeds revealed by genome-wide SNP markers. BMC Genomics. 2017;18:229.

35. Solé M, Gori AS, Faux P, Bertrand A, Farnir F, Gautier M, et al. Age-based partitioning of individual genomic inbreeding levels in Belgian Blue cattle. Genet Sel Evol. 2017;49:92.

36. Yengo L, Zhu Z, Wray NR, Weir BS, Yang J, Robinson MR, et al. Detection and quantification of inbreeding depression for complex traits from SNP data. Proc Natl Acad Sci USA. 2017;114:8602-7.

37. Doekes HP, Veerkamp RF, Bijma P, Hiemstra SJ, Windig JJ. Trends in genome-wide and region-specific genetic diversity in the Dutch-Flemish Holstein-Friesian breeding program from 1986 to 2018. Genet Sel Evol. 2018;50:15

38. Baes CF, Makanjuola BO, Miglior F, Marras G, Howard JT, Fleming $A$, et al. Symposium review: the genomic architecture of inbreeding: How homozygosity affects health and performance. J Dairy Sci. 2019;102:2807-17.

39. Clark DW, Okada Y, Moore KHS, Mason D, Pirastu N, Gandin I, et al. Associations of autozygosity with a broad range of human phenotypes. Nat Commun. 2019;10:4957.

40. Nietlisbach P, Muff S, Reid JM, Whitlock MC, Keller LF. Non-equivalent lethal equivalents: models and inbreeding metrics for unbiased estimation of inbreeding load. Evol Appl. 2018;12:266-79.

41. Alemu SW, Kadri NK, Harland C, Charlier C, Faux P, Caballero A, et al. An evaluation of inbreeding measures using a whole genome sequenced cattle pedigree. Heredity (Edinb). 2020;126:410-23.

42. Caballero A, Villanueva B, Druet T. On the estimation of inbreeding depression using different measures of inbreeding from molecular markers. Evol Appl. 2020;14:416-28.

43. Legarra A, Aguilar I, Colleau JJ. Methods to compute genomic inbreeding for ungenotyped individuals. J Dairy Sci. 2020;103:3363-7.

44. Makanjuola BO, Miglior F, Abdalla EA, Maltecca C, Schenkel FS, Baes CF. Effect of genomic selection on rate of inbreeding and coancestry and effective population size of Holstein and Jersey cattle populations. J Dairy Sci. 2020;103:5183-99.

45. McGivney BA, Han H, Corduf LR, Katz LM, Tozaki T, MacHugh DE, et al. Genomic inbreeding trends, influential sire lines and selection in the global Thoroughbred horse population. Sci Rep. 2020;10:466.

46. Meuwissen THE, Sonesson AK, Gebregiwergis G, Woolliams JA. Management of genetic diversity in the era of genomics. Front Genet. 2020;11:880.

47. Morales-González E, Saura M, Fernández A, Fernández J, Pong-Wong R, Cabaleiro $\mathrm{S}$, et al. Evaluating different genomic coancestry matrices for managing genetic variability in turbot. Aquaculture. 2020;520:734985.

48. Toro MA, Barragán C, Óvilo C, Rodrigáñez J, Rodríguez C, Silió L. Estimation of coancestry in Iberian pigs using molecular markers. Conserv Genet. 2002;3:309-20.

49. Toro MA, Villanueva B, Fernández J. Genomics applied to management strategies in conservation programmes. Livest Sci. 2014;166:48-53.
50. Toro MA, Rodrigañez J, Silió L, Rodríguez MC. Genealogical analysis of a closed herd of black hairless Iberian Pigs. Conserv Biol. 2000;14:1843-51.

51. Weir BS, Cardon LR, Anderson AD, Nielsen DM, Hill WG. Measures of human population structure show heterogeneity among genomic regions. Genome Res. 2005;15:1468-76.

52. Engelsma KA, Veerkamp RF, Calus MPL, Bijma P, Windig JJ. Pedigree- and marker-based methods in the estimation of genetic diversity in small groups of Holstein cattle. J Anim Breed Genet. 2012;129:195-205.

53. Kleinman-Ruiz D, Villanueva B, Fernández J, Toro MA, García-Cortés LA, Rodríguez-Ramilo ST. Intra-chromosomal estimates of inbreeding and coancestry in the Spanish Holstein cattle population. Livest Sci. 2016;185:34-42.

54. Saura M, Tenesa A, Woolliams JA, Fernández A, Villanueva B. Evaluation of the linkage-disequilibrium method for the estimation of effective population size when generations overlap: an empirical case. BMC Genomics. 2015;16:922.

55. Santiago E, Novo I, Pardiñas AF, Saura M, Wang J, Caballero A. Recent demographic history inferred by high-resolution analysis of linkage disequilibrium. Mol Biol Evol. 2020;37:3642-53.

56. Purcell S, Neale B, Todd-Brown $K$, Thomas L, Ferreira MAR, Bender D, et al. PLINK: a tool set for whole-genome association and population-based linkage analyses. Am J Hum Genet. 2007;81:559-75.

57. Kardos M, Nietlisbach P, Hedrick PW. How should we compare different genomic estimates of the strength of inbreeding depression? Proc Natl Acad Sci USA. 2018;115:E2492-3.

58. Henderson CR. Application of linear models in animal breeding. Guelph: University of Guelph Press; 1984.

59. Meuwissen THE, Hayes BJ, Goddard ME. Prediction of total genetic value using genome-wide dense marker maps. Genetics. 2001;157:1819-29.

60. Habier D, Fernando RL, Dekkers JCM. The impact of genetic relationship information on genome-assisted breeding values. Genetics. 2007;177:2389-97.

61. Toro MA, García-Cortés LA, Legarra A. A note on the rationale for estimating genealogical coancestry from molecular markers. Genet Sel Evol. 2011:43:27.

62. Villanueva B, Pong-Wong R, Woolliams JA, Avendaño S. Managing genetic resources in commercial breeding populations. In: Simm G, Villanueva B, Sinclair KD, Townsend S, editors. Farm animal genetic resources. BSAS Occasional Publication No. 30. Nottingham: Nottingham University Press; 2004. p. 113-32.

63. Fernández J, Toro MA, Caballero A. Fixed contributions designs vs. minimization of global coancestry to control inbreeding in small populations. Genetics. 2003;165:885-94.

64. de Cara MAR, Fernandez J, Toro MA, Villanueva B. Using genome-wide information to minimize the loss of diversity in conservation programmes. J Anim Breed Genet. 2011;128:456-64.

65. Gómez-Romano F, Villanueva B, de Cara MAR, Fernandez J. Maintaining genetic diversity using molecular coancestry: the effect of marker density and effective population size. Genet Sel Evol. 2013;45:38.

66. Gómez-Romano F, Villanueva B, Fernández J, Woolliams JA, Pong-Wong R. The use of genomic coancestry matrices in the optimisation of contributions to maintain genetic diversity at specific regions of the genome. Genet Sel Evol. 2016:48:2.

67. Saura M, Pérez-Figueroa A, Fernández J, Toro MA, Caballero A. Preserving population allele frequencies in ex situ conservation programs. Conserv Biol. 2008;22:1277-87.

\section{Publisher's Note}

Springer Nature remains neutral with regard to jurisdictional claims in published maps and institutional affiliations. 